\title{
NÄHE UND FERNE KAISERLICHER MACHT: DAS BEISPIEL KÖLN Von WERNER ECK
}

Die Szene ist bekannt. Eine Gruppe von Pharisäern will nach Lukas 20, $20 \mathrm{ff}$. Jesus entweder vor dem Volk oder der höchsten römischen Provinzautorität, dem praefectus Iudaeae Pontius Pilatus, in Schwierigkeiten bringen; sie fragen ihn deshalb, ob es ihnen, den Juden, erlaubt sei, dem Kaiser Steuern zu zahlen oder nicht ( aber antwortet mit einer Gegenfrage, nachdem man ihm einen Denar gereicht hatte: „Wessen Bild und Aufschrift zeigt er (der Denar)?“

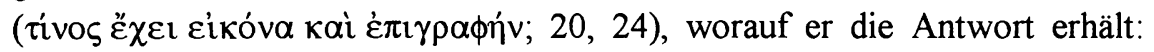

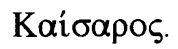

Einfacher, aber auch selbstverständlicher als in dieser Szene könnte man nicht zeigen, wie die politische Macht Roms, verkörpert im Kaiser, in den Provinzen präsent war und worin sie sich ganz selbstverständlich konkretisierte. Für gläubige Juden war es unerträglich, daß Kaiserbilder nach Jerusalem gebracht wurden. Die Präfekten Judäas der frühen Jahre nach der Provinzialisierung ließen deshalb die Bilder des Herrschers von den Feldzeichen nehmen, wenn sie mit ihren Truppen nach Jerusalem hinauf zogen - es sei denn, sie wollen die Bevölkerung provozieren wie Pontius Pilatus dies getan hat. ${ }^{1}$ Doch römische Denare, auf denen generell nicht nur der Name, sondern auch das Bild des Herrschers erschien, waren offensichtlich ganz selbstverständlich auch in den Händen von gläubigen Juden. Es waren ja die Schriftgelehrten und Priester selbst, die Jesus den Denar gereicht hatten. ${ }^{2}$ Der ferne Kaiser war unmittelbar durch sein Bild präsent, jeder konnte eine Vorstellung davon haben, wer derjenige war, der von Rom aus die Welt regierte, dem alle gehorchen und Steuern zahlen mußten. Kein sonstiges Medium der Kommunikation machte den Kaiser so gegenwärtig, so omnipräsent wie die Münzen. Natürlich gab es in allen Städten des Reiches Statuen und Porträts, auch gemalte Bilder, die dem Herrscher Gestalt verliehen, in allen Städten gab es statuarische Darstellungen, oft verbunden mit Inschriften, die Namen und Hoheitstitel verkündeten. ${ }^{3}$ Selbst in Waldgirmes, der zwischen ca. $5 \mathrm{v}$. Chr. und $9 \mathrm{n}$. Chr. an der Lahn

\footnotetext{
${ }^{1}$ Josephus, b. J. 2, 169-171; ant. 14, 65-67.

${ }^{2}$ Lucas 22, 19.

${ }^{3}$ Vgl. nur beispielsweise G. Alföldy, Statuen in Venetia und Histria. Epigraphische Quellen, Abh. Heidelb. Akad. Wiss., Phil-Hist. Kl. 1984, 3, Heidelberg 1984.
} 
aus dem Nichts erbauten Siedlung für dort wohnende Germanen, war bereits eine überlebensgroße bronzene Reiterstatue des Augustus errichtet worden. ${ }^{4}$ Manche dieser Statuen standen auf gewaltigen Bögen hoch über allen Betrachtern; niemand konnte sie übersehen. ${ }^{5}$ Schon in dem erst zwei bis drei Jahrzehnte alten oppidum Ubiorum stand offensichtlich ein solcher Bogen, um den Ruhm des Herrscherhauses an der Rheinfront zu verkünden. ${ }^{6}$ Immer wieder trafen auch Schreiben aus der Zentrale des Reiches ein, durch die der Herrscher mit seinen Untertanen in Kontakt trat. Wer lesen erlernt hatte, konnte die ipsissima verba nachlesen, weil sie zumindest für eine gewisse Zeit in der Stadt öffentlich zugänglich waren, geschrieben vielleicht auf tabulae dealbatae oder gelegentlich auch sofort auf Stein oder Bronze. Aber sie wurden auch öffentlich vorgetragen, im Theater oder auf den öffentlichen Plätzen einer Stadt. Manche dieser Schreiben verbreiteten das Bild des Wohltäters der Menschheit; nicht wenige solcher Briefe sind in epigraphischer Form auf uns gekommen. ${ }^{7}$ Doch häufiger enthielten sie Anordnungen über neue Forderungen gegenüber den Untertanen, oft finanzieller Natur; diese sind jedoch zumeist verloren gegangen; es lohnte sich nicht, sie in dauerhafter Form zu publizieren und so zu bewahren. ${ }^{8}$

Durch all diese Medien war der ferne Kaiser präsent, selbst bis in die kleineren Siedlungen und Dörfer hinein. Denn zumindest die Münzen gelangten überall hin. Dennoch: Der Herrscher blieb weit entfernt, er war

\footnotetext{
${ }^{4}$ A. Becker, Die Ausgrabungen in Lahngau-Waldgirmes 1999. Eine nova colonia aus der Zeit des Kaisers Augustus im Lahntal?, in: Denkmalpflege und Kulturgeschichte. Landesamt für Denkmalpflege in Hessen Heft 2, 1999, 60ff:; ders., Eine römische Stadt an der Lahn?, in: Antike Welt $31,2000,601 \mathrm{ff}$.

${ }^{5}$ W. Eck - G. Foerster, Ein Triumphbogen für Hadrian im Tal von Beth Shean bei Tel Shalem, JRA 12, 1999, 294-313 mit der neueren Literatur.

${ }^{6}$ H. v. Hesberg, Bauteile der frühen Kaiserzeit in Köln. Das Oppidum Ubiorum zur Zeit des Augustus, in: Grabung - Forschung - Präsentation. Festschrift Gundolf Precht, Xantener Berichte 12, Mainz 2002, 13ff., bes. $20 \mathrm{f}$.

${ }^{7}$ Die Masse dieser Texte mit kaiserlichen Schreiben bei J.H. Oliver, Greek Constitutions of Early Roman Emperors from Inscriptions and Papyri, Philadelphia 1989 mit den Ergänzungen bei V. I. Anastasiadis - G. A. Souris, An Index to Imperial Constitutions from Greek Inscriptions and Papyri 27 $\mathrm{BC}$ to $284 \mathrm{AD}$, Berlin 2000, $2 \mathrm{ff}$.

${ }^{8}$ W. Eck, Zur Einleitung. Römische Provinzialadministration und die Erkenntnismöglichkeiten der epigraphischen Überlieferung, in: Lokale Autonomie und römische Ordnungsmacht in den kaiserzeitlichen Provinzen vom 1. bis 3. Jahrhundert, hg. W. Eck, München 1999, 1ff. Zu dem Schreiben des Antoninus Pius an Ephesus (I. Eph. V 1491), das bisher durchwegs als Tadel an der Stadt angesehen wurde und deshalb als einer der wenigen kaiserlichen Briefe mit negativem Inhalt galt, siehe jetzt mit einer neuen Ergänzung und dann positiven Aussage Chr. Kokkinia, Letters of Roman authorities on local dignitaries. The case of Vedius Antoninus, ZPE 142, 2003, $197 \mathrm{ff}$.
} 
kein deus praesens. ${ }^{9}$ Weit konkreter waren da schon die Statthalter, die als seine Vertreter in der Provinz agierten, seine Prokuratoren mit ihrem nicht immer sehr beliebten Personal aus kaiserlichen Sklaven und Freigelassenen und schließlich die Soldaten, die mehr als alle anderen Repräsentanten kaiserlicher Macht überall gegenwärtig waren. ${ }^{10}$ Der Kaiser gehörte vor allem in den Wirkungen, die von ihm ausgingen, zur Normalität des provinzialen Lebens, nicht jedoch seine unmittelbare Gegenwart.

Dennoch gab es die Vorstellung, daß der Herrscher vor Ort erscheinen müsse, daß seine Gegenwart notwendig sei. Geradezu klassisch ist dies im s.c. de $\mathrm{Cn}$. Pisone patre im Jahr $20 \mathrm{n}$.Chr. vom Senat formuliert worden: Germanicus Caesar, qui a principe nostro ex auctoritate huius ordinis ad rerum transmarinarum statum componendum missus esset, desiderantium praesentiam aut ipsius Ti. Caesaris Augusti aut filiorum alterius ipsius. ${ }^{11}$ Tatsächlich hat ja auch Germanicus den Osten des Reiches als Tiberius' Repräsentant besucht. Ähnlich drückt diese Vorstellung Velleius Paterculus an zwei Stellen seines Werkes aus: Hispaniae nunc ipsius Augusti praesentia, nunc Agrippae... multo varioque Marte pacatae ${ }^{12}$ ferner: Caesar circumferens terrarum orbi praesentia sua pacis suae bona ${ }^{13}$ Und schon Horaz hatte für Augustus formuliert: o tutela praesens Italiae dominaeque Romae. ${ }^{14}$ Auch der anonyme Panegyriker, der im Jahr 311 vor Constantin in Trier sprach, weiß um die Vorstellung von der segensreichen Gegenwart des Kaisers, wenn er von den prachtvollen Bauten dieser Stadt sagt: Quae certe omnia sunt praesentiae tuae munera. ${ }^{15}$ Und ein anderer Panegyriker rühmte Trier, quae adhuc adsiduitate praesentiae tuae prae ceteris fruitur. ${ }^{16}$ Mit der unmittelbaren Präsenz des Herrschers sollen Glück und Wohlstand, Sicherheit und Ordnung angekommen sein. Die Menschen warten auf das Erscheinen des Kaisers, was die Griechen als Epiphanie bezeichnen. Die Sphäre zu den Göttern wird berührt.

\footnotetext{
${ }^{9}$ Siehe z.B. AE 1980, 52; vgl. AE 1919, 26; 1920, 23; 1957, 246.

${ }^{10}$ Vgl. z.B. B. Isaac, Army and Power in the Roman World: A Response to Brian Campbell, in: Army and Power in the Ancient World, hg. A. Chaniotis - P. Ducrey, Stuttgart 2002, $181 \mathrm{ff}$.

${ }^{11}$ W. Eck - A. Caballos - F. Fernández, Das senatus consultum de Cn. Pisone patre, München 1996, 40f. Zeile 30ff.; 162.

${ }^{12}$ Velleius Paterculus 2, 90, 1 .

${ }^{13}$ Velleius Paterculus 2, 92, 2.

${ }^{14}$ Horatius, carm. 4, 14, $43 \mathrm{f}$.

${ }^{15}$ Pan. Lat $6(7), 22,5$.

${ }^{16}$ Pan. Lat. $5(8), 2,1$.
} 
Daß solche Sätze zumeist mehr über die Vorstellungen einzelner Rhetoren und Theoretiker als über die Realität aussagen, braucht nicht betont zu werden. Deshalb ist es vielleicht aussagekräftiger, sich an einem konkreten Fall zu vergegenwärtigen, was die Anwesenheit eines Kaisers für eine Provinzstadt wirklich bedeuten konnte - oder vielleicht auch die Abwesenheit. Manche Städte bieten sich für solche Betrachtungen an, etwa Ephesus, Athen, Antiochia in Syrien oder Alexandria in Ägypten. ${ }^{17}$ Sie alle waren Hauptzentren des Imperiums mit überdurchschnittlich großer Bevölkerung und daraus resultierenden Problemen. Vor allem aber waren sie kontinuierlich Ziel auch der Herrscher, soweit sie das Reich bereisten. Doch es sei gestattet, die angesprochene Thematik an einem Exemplum zu betrachten, das mir geographisch und sachlich näher liegt, nämlich an der Colonia Claudia Ara Agrippinensium, dem heutigen Köln. Diese Stadt bietet sich vielleicht auch deswegen an, weil sie vom Beginn der monarchischen Herrschaft in Rom bis fast ans Ende des weströmischen Teils des Reiches, wenn auch mit großen Intervallen, immer wieder von einzelnen Herrschern aufgesucht wurde, in sehr unterschiedlichen politischen und militärischen Situationen. ${ }^{18}$

Daß der zentrale städtische Kern des Stammes der Ubier an der Stelle des heutigen Köln entstand, war eine direkte Folge der von Augustus angeordneten Offensive gegen die rechtsrheinischen Germanen. Topographische Gegebenheiten wie die Rheintalstraße, das hochwasserfreie Plateau und der durch eine vorgelagerte Rheininsel geschaffene natürliche Hafen waren wohl die Voraussetzungen, daß das römische Militär und vor allem der Oberkommandierende der Offensivarmee dort bald öfter Station machten. Die Folge war der durch römische Kräfte betriebene systematische Ausbau der Stadt, nicht nur in der Grundrißplanung, sondern auch der konkreten Ausgestaltung des Ortes mit monumentalen Steinbauten, und zwar noch in augusteischer und frühtiberischer Zeit. Das früheste Monument, das dies zeigt und auch sehr genau datiert werden kann, ist das sogenannte Ubiermonument, vermutlich ein Eckturm der Stadtbefestigung, der gerade nicht auf ubische Initiative zurückgeht, sondern eindeutig von einer römischen Autorität veranlaßt wurde. Allein aus diesem Monument, dem kleinen

\footnotetext{
${ }^{17}$ Siehe zu diesen Städten im Zusammenhang mit Kaiserbesuchen H. Halfmann, Itinera principum. Geschichte und Typologie der Kaiserreisen im Römischen Reich, Stuttgart 1986; auch bei J. Lehnen, Adventus principis. Untersuchungen zu Sinngehalt und Zeremoniell der Kaiserankunft in den Städten des Imperium Romanum, Frankfurt 1997 ist einiges dazu angemerkt.

${ }^{18}$ Siehe dazu im Überblick H. Volkmann, Römische Kaiser in Köln, Gymnasium 1967, 119ff;; ausführlich in Kürze W. Eck, Geschichte der Stadt Köln - Die römische Zeit (in Druckvorbereitung).
} 
Überrest eines größeren Ganzen, ließe sich der kaiserliche Wille ableiten, der hinter der Erbauung dieses städtischen Zentrums, dieser „Stadt aus dem Nichts" stand. ${ }^{19}$ Die Siedlung verfügte offensichtlich nach kurzer Zeit noch unter Augustus nicht nur über Wohnmöglichkeiten, die selbst für den Oberkommandierenden Germanicus und seine Frau Agrippina adäquat waren, sondern auch über öffentliche Monumentalbauten wie Tempel und Portiken. Sogar ein Ehren- oder Triumphbogen muß damals bereits für einen der Träger der politisch-militärischen Macht, also für Augustus, Tiberius oder Germanicus errichtet worden sein. Von all dem sind zwar nur noch jämmerliche Reste in Form von Kapitellen, Säulentrommeln oder Säulenbasen und Gesimsen erhalten. Doch Henner von Hesberg hat zu Recht nochmals auf die an sich banale Erkenntnis verwiesen, daß jedes architektonische Fragment auf etwas ursprünglich Ganzes, auf ein gesamtes Bauwerk verweist. ${ }^{20}$ Die Konsequenz dieser Überlegung ist für Köln, daß dieses schon zu Beginn seiner Existenz sich nicht wie ein germanisches Bauerndorf präsentiert hat, sondern ein auf die Anwesenheit der Träger der höchsten militärischen und politischen Macht ausgestalteter Ort war, der zudem durch den Altar für den provinzialen Herrscherkult der Provinz Germania eine besondere politische Qualität erhielt. Wären aber nicht Tiberius oder Germanicus mit ihrem Stab oder Hof, wie immer man das nennen will, dorthin gekommen und für längere Zeit präsent gewesen, dann hätte sich, nach allem was für uns erkennbar ist, die Siedlung nicht entwickeln können oder zumindest nicht in der Art, wie es dann tatsächlich geschah. Die praesentia dieser Mitglieder der domus Augusta war die Voraussetzung für die Entstehung dieses städtischen Mittelpunkts. Ob Augustus selbst jemals bis an die Stelle des späteren Köln gekommen ist, läßt sich nicht sagen. Aber es ist durchaus möglich, daß er sich während seines langen, von 16-13 v.Chr. dauernden Aufenthalts in den gallischen Provinzen, auch bis in die nördlichen Rheingegenden begeben hat, zumal da in dieser Zeit die Entscheidung über die Eroberung der rechtsrheinischen Gebiete getroffen wurde. Die Präsenz der ersten Truppen unmittelbar an der Rheinfront geht bereits auf die Zeit von Augustus' Präsenz in Gallien zurück.

Die Erhebung des Zentralorts der Ubier zur Kolonie im Jahr 50 vollzog sich allerdings ohne jede persönliche Anwesenheit der beiden Personen, die diese Entscheidung getroffen hatten: Claudius und Agrippina. Dennoch: Wie auch immer die wirklichen aktuellen Motive Agrippinas

\footnotetext{
${ }^{19}$ Siehe vorerst W. Eck, Die Anfänge des römischen Köln. Logistisches Zentrum zur Unterwerfung Germaniens, Kölner Universitätsjournal 32, 2, 2002, 56ff.

${ }^{20}$ Hesberg, Bauteile (Anm. 6) $13 \mathrm{ff}$.
} 
gewesen sein mögen, ohne ihre nur dem Zufall zu verdankende Geburt in dem Vorort der Ubier 35 Jahre früher wäre die Stadt am Rhein nicht das Objekt ihres Ehrgeizes geworden, den eigenen Geburtsort ebenso hervorgehoben zu sehen wie Lugdunum, wo ihr Gatte das Licht der Welt erblickt hatte. ${ }^{21}$ Die Folgen dieses „kurzfristigen Aufenthalts“ Agrippinas für das innere Leben der großen Gemeinde waren grundstürzend: eine politische Einheit, die civitas der Ubier verschwand entweder direkt oder doch bald danach, eine römische Kolonie trat an ihre Stelle. Zahllose Menschen waren von der Entscheidung des Kaisers und seiner Gemahlin, die im fernen Rom gefällt worden war, betroffen. $\mathrm{Ob}$ es darüber Verhandlungen mit Vertretern der Ubier am Kaiserhof gegeben hat, ist nicht überliefert - möglich ist es, wahrscheinlich ist es nicht. Der ferne Herrscher und noch mehr seine Gattin an seiner Seite, sie wußten, was richtig und wichtig war für die Menschen am Rhein.

Dann erlebte die Stadt am 2. Januar 69 die Akklamation des Vitellius durch die Truppen am Rhein, den Ausgangspunkt turbulenter Ereignisse, die das Gemeinwesen in die schlimmste Krise seiner römischen Geschichte vor der Mitte des 3. Jh. stürzten. Für einen Augenblick war die Stadt Mittelpunkt des politisch-militärischen Lebens des Reiches. Manche Bewohner mochten persönliche Hoffnungen damit verbinden. Die Agrippinenser unterstützten wie andere Gemeinden in Gallien den in ihren Mauern weilenden Herrn - was wäre ihnen auch anderes möglich gewesen? Doch die Anwesenheit des neuen Herrschers dauerte nur kurz. Die Folgen freilich trafen die CCAA für die nächsten drei Jahre in besonders harter Form. Es hätte auch das physische Ende der Kolonie sein können. $\mathrm{Zu}$ welchen konkreten Folgen das zufällige Ereignis der Akklamation eines Herrschers in der Stadt geführt hätte, das enthüllte sich nicht mehr, da Vitellius bereits vor Ende des Jahres 69 scheiterte. Wer ihn unterstützt hatte, mußte froh sein, wenn dies vergessen wurde. Schon damals bewahrheitete sich jedoch ein geläufiger Köl'scher Satz, der - auf Hochdeutsch - lautet: „Es ist noch immer alles gut gegangen“.

Knapp 30 Jahre später wurde erneut ein Kaiser in der Stadt ausgerufen, diesmal freilich nicht durch die Truppen gegen den legitimen Herrscher in Rom; vielmehr erhielt Traian Anfang Februar 98 die Nachricht vom Tod seines Adoptivvaters und seiner eigenen Akklamation als Augustus apud

\footnotetext{
${ }^{21}$ Siehe dazu ausführlich W. Eck, Agrippina - die 'Stadtgründerin' Kölns. Eine Frau in der frühkaiserzeitlichen Politik, Köln ${ }^{2}$ 1993, 77ff.; H. Galsterer, Von den Eburonen zu den Agrippinensern. Aspekte der Romanisierung am Rhein, KJ 23, 1990, $117 \mathrm{ff}$.
} 
Agrippinam. ${ }^{22}$ Er hatte kurz vorher, im Oktober 97, durch eine sorgfailtig von Rom und von der Provinz aus inszenierte Pression seine Adoption durch den kinderlosen, mehr als 66 Jahre alten Kaiser Nerva durchgesetzt; später wurde behauptet, Jupiter selbst habe dem regierenden Kaiser diesen Gedanken eingegeben. ${ }^{23}$ Real war nur die militärische Macht, die hinter Traian stand. Diesmal mußte niemand in Köln überlegen, ob es klug sei, diesem neuen Kaiser zuzujubeln. Die Akklamation war unstrittig. Erneut war die Stadt Mittelpunkt der Politik des gesamten Reiches; nicht nur die senatorischen und ritterlichen Amtsträger, die nicht an Rom gebunden waren, eilten an den Rhein; auch die Städte und civitates Italiens und der Provinzen sandten ihre führenden Männer an den Rhein, um den neuen Kaiser zu begrüßen. Köln wurde zu einem Sammelpunkt der Kulturen des Imperiums, und all das nur deswegen, weil der Princeps sich in ihren Mauern aufhielt. ${ }^{24}$ Für manche der Stadtbewohner mochte sich durch die Anwesenheit so vieler zahlungskräftiger Gäste ein sehr konkreter Gewinn ergeben. Auch große Politik erlebte man. Denn Casperius Aelianus, einer der Prätorianerpräfekten, der im Verlauf des Jahres 97 zusammen mit seinen Prätorianern Nerva im Stich gelassen hatte, wurde an den Rhein gerufen und hier kurzerhand beseitigt. ${ }^{25}$ Andererseits kam eine Gesandtschaft des Senats, ehrwürdige Herren im römischen Bürgerkleid, hierher, um Traian als den neuen Herrn zu begrüßen. Köln erlebte die große Welt des Imperium. Geschah aber mehr? Gab es Folgen für die Stadt selbst oder ihre Bewohner? Blieb etwas Dauerhaftes von dieser Anwesenheit Traians, die vermutlich doch einige Monate gewährt hatte? Nach den uns erhaltenen Quellen hat diese praesentia principis zu nichts Dauerhaftem geführt - außer zur Gründung der zweiten colonia am Rhein, der colonia Ulpia Traiana (Xanten). Die Agrippinenses behielten dies wohl eher als nicht so ganz erfreuliche Geste des neuen Herrschers im Gedächtnis. Denn mit dem einmaligen Status der Stadt als einzigem hochprivilegierten Ort in der Provinz Niedergermanien war es nun vorbei. Doch angesichts der Quellenarmut, und

\footnotetext{
${ }^{22}$ Eutropius, breviarium 8,2,2; Epitome de Caesaribus 13,3; Hieronymus, Chronik 275f. Zeile 16 zum Jahr 2113; Orosius 7,12,2; Sidonius Apollinaris, carm. 7,114f.

${ }^{23}$ W. Eck, Traian: Der Weg zum Kaisertum, in: Traian, hg. A. Nünnerich-Asmus, Antike Welt, Sonderband, Mainz 2002, 7ff. sowie ders., An Emperor is Made: Senatorial Politics and Trajan's Adoption by Nerva in 97, in: Philosophy and Power in the Graeco-Roman World: Essays in Honour of Miriam Griffin, hg. Gillian Clark - Tessa Rajak, Oxford 2002, $211 \mathrm{ff}$.

${ }^{24}$ Siehe allgemein zu den Folgen der Anwesenheit eines Herrschers in provinzialen Städten $\mathrm{H}$. Halfmann, Itinera principum. Geschichte und Typologie der Kaiserreisen im Römischen Reich, Stuttgart 1986, 124ff. 151ff.

${ }^{25}$ Cassius Dio 68, 5, 4.
} 
zwar auch bei den epigraphischen und archäologischen Zeugnisse, verbietet es sich, aus dem Schweigen der Quellen etwas abzuleiten. ${ }^{26}$

Nach der Abreise Traians in den Donauraum im Sommer 98 scheint für mehr als 150 Jahre kein Kaiser, aber auch kein Mitglied der kaiserlichen Familie mehr den Weg an den Niederrhein genommen zu haben, außer vermutlich Hadrian. Doch selbst von ihm wissen wir nicht, ob er Köln besucht hat, als er auf seiner Reise nach Britannien durch die gallischen Provinzen zog; dies ist freilich sehr wahrscheinlich; denn weshalb hätte er ausgerechnet die niedergermanische Provinzhauptstadt vermeiden und sie damit desavouieren sollen ${ }^{27}$ Dagegen kam Septimius Severus bei den Kämpfen gegen Clodius Albinus in den Jahren 196/7 kaum bis an den Rhein. Darüber war wohl kaum jemand in der Stadt besonders unglücklich; ein im Bürgerkrieg engagierter Kaiser konnte zu ungewöhnlichen Maßnahmen gezwungen sein.

Niemand in der CCAA war freilich schon zu Beginn des 2. Jh. in der Lage zu erkennen, daß für Köln eine Epoche geendet, daß etwas Neues begonnen hatte. Die Kaiserbesuche hörten auf, die Nicht-praesentia des Kaisers wurde zur Normalität, so wie sie für fast alle provinzialen Städte galt. Damals mochten manche in Köln dies bedauert haben; denn nach den allgemeinen Vorstellungen brachte die Anwesenheit des Kaisers laetitia und gaudium mit sich, auch wenn dies niemals voll zugetroffen hat. ${ }^{28}$ Erst mehr als 150 Jahre später merkte man, welch glücklicher Zustand mit der Abwesenheit, der absentia, des Herrschers verbunden sein konnte, nicht ursächlich, aber als Symptom. Denn als kurz nach der Mitte des 3. Jh. Kaiserbesuche in der CCAA wieder einsetzten, war die Zeit eine andere geworden. Die Kaiserbesuche wurden häufiger, aber von gaudium und laetitia war kaum mehr etwas zu spüren. ${ }^{29}$

\footnotetext{
${ }^{26}$ Man könnte sich beispielsweise vorstellen, daß manche Bewohner der Stadt die Chance ergriffen, vom Kaiser für sich selbst oder einen der Söhne die Aufnahme in den equester ordo zu erbitten. Direkte Zeugnisse aus dieser Zeit gibt es allerdings dafür nicht. Für die spätere Entwicklung, die freilich damals ihren Anfang genommen haben könnte, siehe W. Eck, Ein Kölner in Rom? T. Flavius Constans als kaiserlicher Prätorianerpräfekt, in: Grabung - Forschung - Präsentation. Festschrift Gundolf Precht, Xantener Berichte 12, Mainz 2002, 37ff.

${ }^{27}$ Siehe zu den Reisen Hadrians Halfmann (Anm. 24) 188ff.; ferner A.R. Birley, Hadrian. The restless emperor, London 1997, passim.

${ }^{28}$ Lehnen (Anm. 17) 45.

${ }^{29} \mathrm{Zu}$ dieser Epoche jetzt auch H. Hellenkemper, Köln 260 - 355 A.D. Ein unruhiges Jahrhundert Stadtgeschichte, in: Grabung - Forschung - Präsentation. Festschrift Gundolf Precht, Xantener Berichte 12, Mainz 2002, $43 \mathrm{ff}$.
} 
Der erste gesicherte Besuch eines Herrschers nach Traian erfolgte mehr als 150 Jahre nach dessen Aufenthalt. Durch eine Inschrift aus Aphrodisias in Karien wissen wir, daß nicht nur Gallienus sich in der CCAA aufgehalten hat, sondern auch sein Vater Valerian, was den literarischen Quellen nicht zu entnehmen war. Denn von Agrippina aus hat Valerian einen Brief an diese kleinasiatische Gemeinde gerichtet. ${ }^{30}$ Die Datierung des Briefes ist zwar verloren, doch kann man mit guten Gründen davon ausgehen, Valerian habe sich im Sommer 257 in der niedergermanischen Metropole aufgehalten und dabei den Brief nach dem Osten gesandt. ${ }^{31}$ Warum beide Augusti in diesem Jahr an den Rhein gezogen waren, ist keiner Quelle direkt zu entnehmen; doch angesichts der Gesamtlage des Reiches und der vielen anderen Unruheherde kann dieses Faktum nur heißen, daß die Rheinfront höchst gefährdet war. Ansonsten wäre der Kaiser nicht zusammen mit seinem Sohn nach dem Norden gezogen. Bezeugt sind Durchbrüche von Alamannen an oberer Donau und am Oberrhein. Doch auch die Franken am Niederrhein dürften sich damals deutlich bemerkbar gemacht haben.

Reflex des Aufenthalts der beiden Kaiser sind die Beinamen Valeriana Gallieniana, die die Stadt angenommen hat. ${ }^{32}$ Sie sind noch heute auf dem Bogen des Nordtores, der im Römisch-Germanischen Museum wieder aufgestellt ist, zu lesen. Sie sind vielleicht auch mit einer partiellen Erneuerung und Ausbesserung der Stadtmauer verbunden, nicht jedoch mit einem Neubau, wie dies gelegentlich behauptet wurde. Noch wichtiger ist jedoch, daß damals aus dem an der Donau gelegenen Viminacium ein Teil des Personals der dortigen Münzstätte nach Köln verlegt wurde. ${ }^{33}$ Das geschah vielleicht schon im Jahr 256; auf jeden Fall kann dies nur heißen, daß die Kaiser davon ausgingen, am Rhein werde in der nahen Zukunft ein Schwerpunkt der kriegerischen Auseinandersetzungen liegen, weshalb eine Münzstätte notwendig sei, die die Truppen kontinuierlich mit Geld versorgen würde.

Valerian verließ die Stadt bald wieder. Doch zumindest Gallienus muß über längere Zeit sein Hauptquartier in der CCAA aufgeschlagen haben. Dazu bestand offensichtlich auch aller Grund. Denn im Jahr 258 oder

\footnotetext{
${ }^{30} \mathrm{~J}$. Reynolds, in: Ch. Roueché, Aphrodisias in Late Antiquity, London 1989, $1 \mathrm{ff}$.

${ }^{31} \mathrm{M}$. Christol, Les déplacements du collège impérial de 256 à 258: Cologne, capitale impériale, Cahiers du Centre Gustave Glotz 8, 1997, 243ff.

${ }^{32}$ CIL XIII 8261 = B. und H. Galsterer, Die römischen Steininschriften aus Köln, Köln 1975, Nr. 184.

${ }^{33}$ G. Elmer, Die Münzprägung der gallischen Kaiser in Köln, Trier und Mailand, BJ 146, 1941, lff.; M. Christol, Effort de guerre et ateliers monétaires de la périphérie au III siècle apr. J.-C. L' atelier de Cologne sous Valérien et Gallien, in: Armées et fiscalité dans le monde antique, Paris 1977, 235ff.
} 
259 scheint es zu massiven Einbrüchen von Reichsfeinden an der Rheinfront gekommen zu sein, trotz der Anwesenheit des Herrschers. Zwar gibt es dafür kaum vertrauenswürdige schriftliche Quellen, obwohl der spätantike Autor Aurelius Victor behauptet, fränkische Scharen seien durch ganz Gallien bis nach Tarragona in Spanien vorgedrungen ${ }^{34}$ Doch es gibt objektivere Quellen, die eine Aussage erlauben. Denn im gesamten Gebiet westlich von Köln bis weit nach Belgien hinein haben sich zahlreiche Münzhorte gefunden, deren Schlußmünzen in das Jahr 258 oder 259 gehören. Solche Münzschätze wurden z. B. in Erftstadt, Euskirchen, Xanten, Mürlenbach (Kreis Daun) und vor allem in Niederbieber aufgedeckt. ${ }^{35}$ Obwohl derartige Münzkomplexe aus sehr verschiedenartigen Gründen vergraben worden sein können, ${ }^{36}$ läßt die zeitliche Massierung in den Jahren 258/9 und auch die Zusammensetzung mit größter Wahrscheinlichkeit nicht mehr an einzelne sehr individuelle Gründe denken, die zur Verbergung im Boden geführt haben. Vielmehr weisen sie auf ein weitgespanntes, katastrophales kriegerisches Ereignis hin, das auch die Umgebung von Köln getroffen haben muß. Diesem Einbruch von Germanenscharen, der nicht vor dem Jahre 259 erfolgt sein kann, vielleicht sogar erst im Jahr 260, sind aber offensichtlich schon deutliche Spannungen vorausgegangen, die es wohl verständlich machen, daß Gallienus für einige Zeit seinen Sitz am Rhein genommen hat.

Doch andere Katastrophen zwangen ihn, die Rheinfront zu verlassen. Seinen Sohn Saloninus ließ er in Köln als seinen Vertreter zurück. Schon im Jahr 258 hatte dieser den Caesartitel erhalten, womit er als Vertreter der kaiserlichen Macht auftreten konnte. Köln blieb sein Zentrum, obwohl ihm alle gallischen Provinzen untergeordnet waren. ${ }^{37}$ Das kann nur heißen, daß an diesem Teil der Grenze auch die größte Gefahr drohte. Was später Diocletian systematisch betrieb, nämlich, größere Provinzkomplexe durch die Anwesenheit eines Mitglieds des Kaiserkollegiums zu stärken, das deutet

\footnotetext{
${ }^{34}$ Aurelius Victor, Caes. 33, 1.

${ }^{35}$ Siehe das Material bei H.J. Schulzki, Der Katastrophenhorizont der zweiten Hälfte des 3. Jahrhunderts. Historisches Phänomen und numismatischer Befund, KJb 34, 2001, 7ff.

${ }^{36} \mathrm{Vgl}$. K. Strobel, Pseudophänomene der römischen Militär- und Provinzgeschichte am Beispiel des „Falles“ des obergermanisch-raetischen Limes. Neue Ansätze zu einer Geschichte der Jahrzehnte nach 253 n. Chr. an Rhein und oberer Donau, in: Roman Frontier Studies 17, 1997, Zalau 1999, 9ff., der darauf zu Recht hinweist; doch selbst wenn beispielsweise die Befunde von Niederbieber eher mit den inneren Unruhen erklärt werden müssen, kann dies bei den anderen Münzfunden auf der linken Rheinseite kaum der Fall sein.

${ }^{37}$ Dies darf man vielleicht aus AE 1971, 23, einer Weihung der tres provinciae Galliae an Saloninus in Rom, entnehmen.
} 
sich hier bereits an. So mochte man sich in der niedergermanischen Hauptstadt damit trösten, daß die Präsenz des Kaisersohnes eine Gewähr gegen äußere Gefahren bot.

Allgemeine Sicherheit garantierte sie dennoch nicht, wie sich bald zeigte. Denn die eigentliche militärische Macht und der Zugriff auf die Truppen in der Germania inferior lag in der Hand eines anderen, des Marcus Cassianius Latinius Postumus. Ob er dort als Statthalter fungierte oder als außerordentlicher Heereskommandeur, läßt sich nicht sicher entscheiden. Am wahrscheinlichsten ist immer noch, daß er senatorischer Legat in Niedergermanien war. Er rebellierte schließlich gegen Saloninus, als der junge Herrscher von Postumus' Truppen die Herausgabe der Beute verlangte, die sie eben räuberischen Germanenscharen abgenommen hatten. Hätte Postumus dem Verlangen zugestimmt, wäre er als erster ein toter Mann gewesen. Postumus zog mit seinen Truppen gegen Köln, wo Saloninus seinen Sitz hatte, und begann die Belagerung. ${ }^{38}$ Soweit wir wissen, war dies die erste richtige Belagerung durch feindliche Truppen, die die Stadt bisher erlebt hatte. Nur die Situation des Jahres 69/70, als die Germanen und Bataver vor den Mauern lagen, war in etwa vergleichbar. In dieser höchst gefährlichen Situation ließ sich Saloninus noch selbst zum Augustus, also zum gleichberechtigten Kaiser neben seinem Vater ausrufen, vielleicht in dem Glauben, so größere Autorität zu gewinnen und sich dadurch bei den Truppen doch noch durchsetzen zu können. $\mathrm{Ob}$ der junge Kaiser sich hätte länger halten können, läßt sich nicht sagen; denn Postumus' Truppen waren natürlich auch in der Belagerungstechnik geschult und hatten die notwendige Ausrüstung zur Verfügung. Doch vielleicht ist es gar nicht zu einer wirklich längeren Belagerung gekommen. ${ }^{39}$ Auf jeden Fall geschah die Entscheidung von innen her. Nach Zosimus ${ }^{40}$ übergaben Saloninus' Soldaten die Stadt an Postumus, weil die Folgen der Belagerung sich bemerkbar machten. Nach Zonaras, einem byzantinischen Autor der 1 . Hälfte des 12 . Jh., übergaben diejenigen, die in der Stadt weilten, Köln an den Angreifer. ${ }^{41}$ Das kann sich auch auf die Agrippinenses selbst beziehen, also die Stadtbewohner im Gegensatz zum militärischen und administrativen Personal um Saloninus.

\footnotetext{
${ }^{38}$ Zosimus 1, 38, 2.

39 Allerdings sollte man dies auch nicht ausschließen; immerhin könnte die Erneuerung der Stadtmauer, die im 3. Jh. an manchen Stellen von Grund aus erfolgte, eine Folge einer Belagerung und Zerstörung einzelner Teile der Mauer gewesen sein.

${ }^{40}$ Siehe Anm. 38.

${ }^{41}$ Zonaras 12, 24.
} 
Undenkbar ist das nicht; denn vor allem die Interessen der Kölner Führungsschicht reichten über den Stadtkern hinaus. Vor den Mauern lagen ihre Güter und Betriebe, die dem Zugriff der Belagerer ausgeliefert waren. Zudem waren sie vielleicht ebenfalls schon von der Vorstellung ergriffen, $\mathrm{da} ß$ unter den Bedingungen der kontinuierlichen Bedrohung von außen ein naher, mit der Region stärker verbundener Kaiser größeren Vorteil versprach als nur der Sohn des regierenden Kaisers Gallienus, selbst wenn dieser inzwischen selbst zum Augustus avanciert war. Vermutlich hatte man auch schon von der Katastrophe des älteren Augustus Valerianus im Osten gehört. Wie auch immer das gewesen sein mag: Wohl im Hochsommer 260 wurden Kölns Tore geöffnet, Saloninus und sein Berater ausgeliefert; Postumus ließ beide umbringen. ${ }^{42}$ Es war der erste Kaisermord, den die Stadt erlebte.

Für mehr als das nächste Jahrzehnt war die CCAA der Mittelpunkt eines eigenen Reiches. ${ }^{43}$ Die Münzstätte blieb bis Anfang der 70er Jahre in der Stadt. Und notwendigerweise kehrte Postumus so wie auch seine rheinischen Nachfolger Victorinus und Tetricus immer wieder in diese Stadt zurück. Münzen verweisen auf den processus consularis; so liegt es nicht allzu fern anzunehmen, die verschiedenen Kaiser hätten ihre Konsulate in ihrem Zentrum angetreten. ${ }^{44}$ Panegyriker, die wir auch in der Umgebung dieser gallischen Gegenkaiser vermuten dürfen, konnten darin schwelgen, die glücklichen Folgen der praesentia Augusti in der niedergermanischen Kolonie zu beschreiben, ähnlich wie dies der Panegyriker von 311 getan hat, als er die Folgen der Entscheidung Constantins furr Trier geschildert hat. Er spricht vom Palatium, den Thermen, den Portiken, quae certe omnia sunt praesentiae tuae munera. ${ }^{45}$ Die Rede eines Panegyrikers der Zeit des Postumus ist uns nicht erhalten. Ein Redner hätte allerdings wohl auch Schwierigkeiten gehabt, im Angesicht von Bürgern der Stadt von solch willkommenen Folgen der kaiserlichen Präsenz zu sprechen. Denn zumindest die Ergebnisse der archäologischen Forschung lassen auch nicht im Ansatz erkennen, daß die Präsenz dieser gallischen Kaiser erfreuliche Auswirkungen für das Erscheinungsbild der Stadt gehabt hätte, etwa durch neue, monumentale Bauwerke. ${ }^{46}$ Lediglich an den Mauern lassen sich Erneuerungen nachweisen, die jedoch auch nicht

\footnotetext{
${ }^{42}$ Zosimus 1, 38; Zonaras 12, 24.

${ }^{43}$ Dazu I. König, Die gallischen Usurpatoren von Postumus bis Tetricus, München 1981.

${ }^{44}$ Vgl. J.F. Drinkwater, The Gallic empire, Stuttgart 1987, 174. 185. 186.

${ }^{45}$ Pan. Lat 6 (7), 22, 5.

${ }^{46}$ G. Hellenkemper Salies, Hofkunst in der Provinz? Zur Denkmalüberlieferung aus der Zeit des Gallischen Sonderreiches, BJb 184, 1984, 67ff.
} 
zwingend der Anwesenheit der Herrscher verdankt werden müssen. Was also hätte die Präsenz der Herrscher für mehr als 10 Jahre für die Bewohner der Stadt bedeuten können? Erschien sie nur als unwillkommene Last, die durch Einquartierung und stetige Einforderung von Steuern spürbar war? Vermutlich hätte doch etwas anderes im Vordergrund gestanden, eine Vorstellung, wie sie Ammianus Marcellinus im Jahr 365 die Berater Valentinians I. formulieren ließ. Denn als der Kaiser in diesem Jahr nach dem Osten aufbrechen wollte, um seinem Bruder Valens gegen den Usurpator Procopius zu Hilfe zu eilen, wiesen sie ihn darauf hin, er solle nicht die westlichen Provinzen verlassen und sie so dem Ansturm der Barbaren preisgeben. Gesandte der Städte Galliens seien zu ihnen gekommen und hätten flehentlich gebeten, ne in rebus duris et dubiis impropugnatas eas relinqueret, quas praesens eripere poterit discriminibus maximis, metu ambitiosi nominis sui Germanis incusso. ${ }^{47}$ Valentinians Name also hat sich den Germanen als furchtbar eingegraben, doch dies nur deswegen, weil er persönlich in Gallien anwesend war. Das Itinerar, das sich aus den Propositionsvermerken der von Valentinian erlassenen Gesetze rekonstruieren läßt, zeigt auch, daß er sich kontinuierlich weitgehend in Gallien aufgehalten hat. ${ }^{48}$ Von da her gewinnt die Aussage Ammians auch für die Zeit der gallischen Regionalkaiser reale Substanz, eine Substanz, wie sie später in geradezu stereotyper Weise auch bei den gallischen Panegyrikern immer wieder aufscheint. ${ }^{49}$ Umgekehrt veranlaßt die absentia des Kaisers die Barbaren, erneut anzugreifen, wie etwa die Könige der Francia, qui per absentiam patris tui (nämlich des Constantius Chlorus) pacem violaverant, wie ein Panegyriker gegenüber Constantin betont. ${ }^{50}$ Solches Denken und Fühlen kann man bei den Bewohnern der Grenzstadt Köln sogar in besonderem voraussetzen - und vielleicht auch eine Portion Dankbarkeit gegenüber denen, die aus der Sicht Roms und der jeweils vom Senat akzeptierten Kaiser nur Rebellen in Gallien waren.

Die Situation der Bedrohung verminderte sich zwar an dieser Grenze gegen Ende des 3. Jh., aber grundlegend veränderte sie sich nicht mehr. So blieb auch dieses Gefühl der Erleichterung, der stärkeren Sicherheit bei der Anwesenheit des Kaisers. Deutlich wird dies nochmals besonders bei Constantin, der in den ersten Jahren seiner Regierung fast in jedem Jahr am

\footnotetext{
${ }^{47}$ Ammianus Marcellinus 26, 5, 12.

${ }^{48}$ O. Seeck, Regesten der Kaiser und Päpste für die Jahre 311 bis 476 n.Chr., Stuttgart 1919, 226ff.

${ }^{49}$ R. Beck, Die „Tres Galliae“ und das „Imperium“ im 4. Jahrhundert, Zürich 1969; darin Kap. III: Die gallischen Panegyriker und die Kaiserpräsenz, S. 96ff.

${ }^{50}$ Pan. Lat. $6(7), 10,2$.
} 
Niederrhein und auch in der Colonia Agrippina erschien. ${ }^{51}$ Schon 308 kämpfte er am Niederrhein gegen Frankenkönige, die er dann allerdings nicht in Köln, sondern in Trier im Amphitheater zur Abschreckung für andere Reichsfeinde vor einem großem Publikum hinschlachten ließ. ${ }^{52}$ Spätestens 310 muß er sodann den Bau der Brücke von der Stadt Köln zum gegenüberliegenden Kastell Divitia, der einzigen festen Brückenanlage am Niederrhein, angeordnet haben. ${ }^{53}$ Vollendet wurde diese Anlage aber wohl erst später. Zumindest das castrum selbst wird wohl erst gegen 315 fertig gestellt gewesen sein; denn die Bauinschrift, die nur durch die Überlieferung in der Vita des Abtes Heribert von Deutz auf uns gekommen ist, verweist bereits auf die Decennalien und die vota für die Vicennalien. Noch wichtiger aber ist, daß in dieser Bauinschrift, in der auch die Soldaten der zweiundzwanzigsten Legion als ausführende Kräfte genannt werden, gerade auf die Anwesenheit des Herrschers verwiesen wird; denn der Text erwähnt, der Bau sei durchgeführt worden, nachdem virtute domini Constantini Maximi Pii Felicissimi Invicti Augusti die Franken in ihrem eigenen Land besiegt worden seien. Dann aber folgt etwas, was in einer Bauinschrift höchst auffällig ist: der Bau sei erfolgt sub praesentia principis sui. ${ }^{54}$ Auch im militärischen Kontext kann also diese Vorstellung ihre Wirksamkeit entfalten. ${ }^{55}$ Das hieß selbstverständlich nicht, der Herrscher sei während der gesamten mehr als fünfjährigen Bauzeit des Kastells in Köln gewesen; vielmehr hat Constantin während dieser Jahre fast alle Provinzen seines Reichsteils besucht. Aber der Bau erfolgte nicht durch irgend jemanden aus der Militärhierarchie, sondern auf persönliche Anordnung des Kaisers. Dies alles war so wichtig, daß die Fertigstellung des Deutzer Kastells am Rhein sogar in prunkhafter Form auf einem Goldmultiplum denen mitgeteilt wurde, die besonders an der Verteidigung des Reiches Anteil hatten: den kaiserlichen Truppen. ${ }^{56}$ Denn die Münze, die zur Verteilung an die Truppen bestimmt war und früher auf die Erneuerung der Trierer Moselbrücke bezogen wurde, bezeugt den Bau des Deutzer Kastells mitsamt der zugehörigen Brücke. In der

\footnotetext{
${ }^{51}$ Siehe zu den Daten Seeck (Anm. 48) 159ff.; T. D. Barnes, The New Empire of Diocletian and Constantine, Cambridge 1982, $47 \mathrm{ff}$.

${ }^{52}$ Pan. Lat. 6 (7), 10ff.; 4 (10), 16, 5f. Vgl. Prosopography of the Later Roman Empire I, 1971, 113. 599.

${ }_{53}^{53}$ Pan. Lat. 6 (7), 13.

${ }^{54}$ CIL XIII $8502=$ Dessau $8937=$ Galsterer (Anm. 32) Nr. 187.

${ }_{55} \mathrm{Vgl}$. auch Synesius, de regno 19.

${ }^{56}$ M. R.-Alfolddi, Das Trierer Stadtbild auf Constantins Goldmultiplum: ein Jahrhundertirrtum, in: dies., Gloria Romanorum. Schriften zur Spätantike, hg. H. Bellen - H.-M. v. Kaenel, Stutt. 2001, 143ff.
} 
Mitte des Geschehens aber steht, auch in dem Bild auf der Münze, der Kaiser persönlich.

Der häufige Aufenthalt Constantins in Köln hatte aber offensichtlich noch andere Folgen für die Stadt, für eine bestimmte städtische Gruppe und vielleicht auch fur ihn selbst. Denn als Ende 312 sich die Donatisten aus Africa um die Klärung ihrer Vorwürfe gegen den von ihnen nicht anerkannten Bischof von Carthago, Caecilianus, an Constantin wandten, bestimmte er Bischof Miltiades von Rom als Richter, ordnete ihm aber gleichzeitig noch drei weitere Bischöfe als Beisitzer zu, und zwar drei Bischöfe aus Gallien und Germanien: Reticius von Augustodunum (Autun) ${ }^{57}$ Marinus von Arelate (Arles) und Maternus von Agrippina, eben Köln. ${ }^{58}$ An allen drei Orten hatte sich Constantin in den Jahren vor 312 aufgehalten. ${ }^{59}$ Die Wahl dieser Bischöfe kann kein Zufall gewesen sein; vielmehr muß man wohl schließen, daß Constantin selbst (und vielleicht auch hochrangige Berater aus seiner Umgebung) mit diesen lokalen Leitern christlicher Gemeinden schon vorher zusammengetroffen ist und sie als herausragende Führungspersönlichkeiten erkannt hat. Deshalb konnte auch seine Wahl speziell auf sie fallen und nicht auf Bischöfe italischer Städte, wie es aus der Situation heraus, in der er seine Entscheidung in Italien zu treffen hatte, nahe gelegen hätte.

Constantin hat also wohl in den entsprechenden Städten während seiner verschiedenen Aufenthalte nicht nur mit den üblichen honorati der politischen Gemeinden Verbindung aufgenommen, sondern wohl auch mit den Führern der christlichen Gemeinden. Macht man sich dies klar, dann erklärt es sich auch weit einfacher, weshalb es vor der Schlacht an der Milvischen Brücke gegen Maxentius zu der Entscheidung für den Christengott gekommen ist. Der Kaiser hatte offensichtlich durch den Kontakt zu den christlichen Gemeindeleitern Kenntnisse über diesen neuen Kult erhalten, die seinen Vorstellungen von Politik und Religion entgegen kamen und bald darauf ihre Wirkung entfalten konnten. Hosius v. Corduba ist vermutlich doch nicht der einzige christliche Berater Constantins gewesen, sondern vor allem gallische Bischöfe, darunter Maternus von Köln. Für die christliche Gemeinde in Köln könnte die Bekanntschaft ihres "Hirten" mit dem Herrscher des Westens durchaus Vorteile gebracht haben.

\footnotetext{
${ }^{57}$ Hieronymus, vir. ill. 82; ep. 5, 2. 37.

${ }^{58}$ Euseb., h. e. 10, 5, 19f; Optatus v. Mileve 1,23.

${ }^{59}$ Siehe Barnes (Anm. 51) 68ff.
} 
Die Anwesenheit der Herrscher in Köln läßt sich fast durch das gesamte 4. Jh. hindurch verfolgen. Crispus, Constantins Sohn, weilte am Niederrhein, ebenso Constantins andere Söhne, Constantinus II. und Constans. ${ }^{60}$ Anlaß war fast stets eine militärische Bedrohung durch Stämme im Vorfeld des Reiches, unter denen verschiedene Teilstämme der Franken die aktivsten waren. Schon damals gelang aber kein auf längere Zeit fortwirkender überzeugender Sieg. Die Anwesenheit war fast stets ein Zeichen für eine aktive Gefährdung der Reichsgrenze. Die Präsenz der kaiserlichen Macht war ambivalent.

Köln wurde sogar nochmals Schauplatz einer Usurpation, die freilich wegen ihrer mehr als kurzen Dauer selbst keine wesentlichen Folgen für die Stadt nach sich ziehen konnte. Die wirkliche Gefahr kam von außen. 355 hatte in der Hauptstadt der Germania secunda, wie die Provinz damals genannt wurde, der Heermeister Silvanus das Kommando über alle römischen Truppenverbände in Gallien, eine nominell nicht geringe Machtstellung; allerdings hatte die Schlacht bei Mursa im Jahr 351 gewaltige Lücken im gallischen Heer, das Magnentius in den Donauraum geführt hatte, hinterlassen. Silvanus selbst war fränkischer Herkunf, jedoch bereits im Reich aufgewachsen. Dennoch benutzten seine Gegner auch seine ethnische Herkunft als Argument gegen ihn. Nach Ammianus Marcellinus intrigierten am Hof Constantius II. nach dem Sieg über Magnentius eine Reihe von hochgestellten Amtsträgern gegen Silvanus, angeblich, weil man ihm, der schnell seinen Aufstieg gemacht hatte, die militärische Machtposition nicht gönnte. Man verfaßte gefälschte Briefe, die jedoch zunächst wie echt wirkten, weil sie Silvanus' richtige Unterschrift trugen; man hatte den ursprünglichen Text mit Ausnahme der Unterschrift ausgewaschen. Aus diesem gefälschten Schreiben aber konnte man auf die Vorbereitung einer Usurpation rückschließen, wenn man nur genügend Mißtrauen gegen hohe Amtsträger in sich trug. Das soll bei Constantius II. generell der Fall gewesen sein. Obwohl der Schwindel schließlich aufflog, war Silvanus schon über die Intrigen und die Gefahr, die ihm daraus drohte, informiert; er schloß aus der Erfahrung, die er bisher mit Constantius II. gemacht hatte, daß ein einmal gegen eine bestimmte Person gefaßter Verdacht zwingend Folgen haben würde. Das brachte ihn, so die Überlieferung, schließlich dazu, sich der Bedrohung durch die Akklamation zum Kaiser in Köln zu

${ }^{60}$ C. Th. 3, 5, 7 (Juni oder Juli 345). 
entziehen. ${ }^{61}$ Das war im Spätsommer des Jahres $355 .^{62}$ Wenn die Nachricht, die Usurpation habe insgesamt nur etwa 28 Tage gedauert, zutrifft, ${ }^{63}$ muß die Nachricht von der Erhebung aus Köln sehr schnell nach Mailand gelangt sein, wo sich Constantius II. aufhielt. Er beauftragte sogleich einen anderen Militär, Ursicinus, damit, sich nach Köln zu begeben, um mit der Usurpation fertig zu werden. In Eilritten gelangte dieser in die Germania secunda, nur von wenig mehr als zehn Leuten begleitet, zu denen auch der Historiker Ammianus Marcellinus gehörte, dem wir einen relativ ausführlichen Bericht verdanken ${ }^{64}$ Ursicinus hatte, so Ammianus, gehofft, in Köln anzukommen, bevor man dort wiederum wußte, daß der Kaiser in Mailand bereits informiert war. Das hätte es den Ankömmlingen angeblich erlaubt, als eine normale Gesandtschaft $\mathrm{zu}$ erscheinen, die lediglich die Nachricht überbringen sollte, Silvanus werde durch Ursicinus abgelöst; Silvanus selbst aber solle sich zu weiterer Verwendung nach Mailand begeben. Doch das Gerücht war schneller als die kleine Reisegruppe. So war Silvanus auf der Hut, in der Stadt hatte er zudem zahlreiche Truppen in der Stadt zusammengezogen. In der damaligen Bedrohung durch die Franken konnte das für die Bewohner eine beruhigendes Gefühl verbreiten. Durch diese Vorsichtsmaßnahmen des Silvanus war Ursicinus gezwungen, sich zunächst so zu geben, als ob er mit Silvanus gemeinsame Sache machen wolle. Das schien deswegen durchaus plausibel, weil auch Ursicinus in einem ähnlich gespannten Verhältnis zum Kaiserhof stand wie der Usurpator. Auf diese Weise konnte Ursicinus seine Pläne verheimlichen und sie dann doch ins Werk setzen. Durch Bestechung einiger Truppenteile, der sogenannten bracchiati und cornuti, die offensichtlich direkt in Köln einquartiert waren, gelang es schließlich, Silvanus zu beseitigen. Am frühen Morgen, wohl im September, ${ }^{65}$ brachen sie, wie Ammianus sagt, in die regia ein, was nach allem, was wir wissen, nur das Praetorium in Köln meinen kann, und suchten Silvanus. Doch dieser war nicht dort, wo man ihn vermutet hatte, vielmehr

\footnotetext{
${ }^{61}$ Ammianus Marcellinus 15, 5, $1 \mathrm{ff}$.

${ }^{62}$ Als Datum findet man fast überall den 11. August genannt; das geht auf eine Berechnung von $\mathrm{O}$. Seeck, Zur Chronologie und Quellenkunde des Ammianus Marcellinus, Hermes 41, 1906, 481ff., bes. 501 zurück; vgl. ders., Geschichte des Untergangs der antiken Welt, Stuttgart 1922 (ND 1966), IV 231 und 466f. zurück. Doch enthält diese Berechnung zu viele Unbekannte, als daß man sie als gesichert ansehen dürte.

${ }^{63}$ Aurelius Victor, Caes. 42, 16; Epitome de Caes. 42, 10; Eutrop, breviarium 10, 13.

${ }^{64}$ Andere kurze Berichte bei Aurelius Victor, Caes. 42, 16; Sozomenus, historia ecclesiastica 4, 7, 4; Socrates, historia ecclesiastica 2, 32, 11; Iulian, oratio 1, $48 \mathrm{~b} / \mathrm{c}$. Wichtig ist auch Zonaras 13, 9, 22-24.

${ }^{65}$ Es soll der 7. September gewesen sein; das genaue Datum wiederum nach Seeck (Anm. 48) 232.
} 
gerade auf dem Weg in ein christliches Kultgebäude (ein conventiculum ritus Christiani in den Worten des Ammianus). Er flüchtete zwar noch in eine aedicula, in ein kleines Haus, das auf dem Weg zu der Kirche lag. ${ }^{66}$ Doch es nützte ihm nichts. Die Häscher streckten ihn nieder ${ }^{67}$ Einige der zivilen und militärischen Helfer des Silvanus wurden gefangen genommen, vermutlich nach Italien geschafft und dort hingerichtet. ${ }^{68}$

So lautet der Bericht Ammians über den Usurpationsversuch des Silvanus und seine Beseitigung. Er befriedigt in vielfacher Hinsicht nicht. ${ }^{69}$ Denn vor allem die Vorbereitungen des Silvanus für seine eigene Proklamation muten dilettantisch an und auch das Vorgehen des Ursicinus, in dessen Umgebung Ammianus die Ermordung des Usurpators miterlebt und auch mitvorbereitet hatte, erscheint wenig durchsichtig. Deshalb sind verschiedene Versuche unternommen worden, die Überlieferung anders zu verstehen. Man hat sogar angenommen, Silvanus habe in Wirklichkeit überhaupt keinen Usurpationsversuch unternommen, vielmehr habe Ursicinus aus Gründen der Politik am Hof Silvanus umgebracht, weil sich bestimmte Pläne andernfalls nicht verwirklichen ließen. Ammianus habe dann die gewundene Erzählung erfunden, um Ursicinus' Handeln - und damit auch sein eigenes - zu erklären oder besser zu beschönigen. Diese Erklärung scheitert freilich daran, daß schon kurz nach dem Ereignis und lange vor Ammianus berichtet wird, Silvanus habe sich gegen Constantius empört. ${ }^{70}$ So ist man zuletzt doch wieder zu der Ansicht gekommen, Silvanus habe tatsächlich diesen Versuch gemacht. Allerdings dürfe man sich Silvanus nicht

\footnotetext{
${ }^{66}$ Conventiculum kann keineswegs als kleines Gebäude für den christlichen Kult verstanden werden, weil -iculum nicht notwendig eine Verkleinerung signalisiert, vgl. auch J. Kremer, Studien zum frühen Christentum in Niedergermanien, Diss. Bonn 1993, 114; ebensowenig bedeutet aedicula Kapelle, sondern ein kleines Gebäude unterschiedlicher Form. Der christliche Kontext hat meist dazu gefürt, daß die -mögliche- Bedeutung „Kapelle“ als selbstverständlich angesehen wurde.

${ }^{67}$ Ammianus Marcellinus 15, 5, 24-31; wie die Lücke in 5, 5, 30 genauer zu füllen ist, läßt sich nicht erkennen.

${ }^{68}$ Ammianus Marcellinus 15, 6.

${ }^{69}$ Siehe zuletzt vor allem U. Süßenbach, Das Ende des Silvanus in Köln. Statthalterpalast, Fahnenheiligtum und Bischofskirche im frühchristlichen Köln. Eine Bemerkung des Ammianus Marcellinus zum Jahr 355, in: Jahrbuch des Köln. Geschichtsvereins 55, 1984, lff.; J. F. Drinkwater, Silvanus, Ursicinus and Ammianus: Fact or Fiction?, in: Studies in Latin Literature and Roman History 7 , hg. C. Deroux, Brüssel 1994, 569ff; B. Bleckmann, Silvanus und seine Anhänger in Italien: Zur Deutung zweier kampanischer Inschriften für den Usurpator Silvanus (CL X 6945 und 6946), Athenaeum 88, 2000, 477ff.; ferner R. Syme, Ammianus and the Historia Augusta, Oxford 1968, 53ff. und T. D. Bames, Ammianus Marcellinus and the Representation of Historical Reality, Ithaca 1998, 18f.

${ }^{70}$ So Aurelius Victor, Caes. 42, 14ff. in seinem kleinen Geschichtsabriß aus dem Jahr 360 sowie bei Kaiser Julian in seinem Brief an die Athener aus dem Jahr 361 (or. 1, 48 b/c).
} 
als einen barbarischen Franken vorstellen, der im römischen Milieu keinen Rückhalt gefunden habe; vielmehr müsse man die Hinweise in der Historiographie beachten, Silvanus sei, trotz seiner Herkunft, ein kultivierter Mann gewesen, der auch zu alten Familien in Italien Beziehungen gehabt habe. Damit würde der Usurpation doch ein weit ernsterer Hintergrund zugewiesen. $^{71}$ Wenn man freilich von diesem Blickwinkel aus auf die Usurpation blickt, dann kann es sich nicht mehr nur um eine aus dem militärischen Milieu geborene Angelegenheit handeln, dann waren auch zivile Kreise darin eingebunden. Warum nicht auch Bewohner Kölns?

Wenn das so war, dann hat dies allerdings nach der Beseitigung des Silvanus wenig Konsequenzen nach sich gezogen. Denn kurze Zeit nach dem Ende des Aufstandsversuchs, vielleicht sogar als Folge des gescheiterten Aufstands, brach über die Stadt eine Katastrophe herein, wie man sie bisher nicht erlebt, ja früher nicht einmal für möglich gehalten hatte: die Stadt wurde erstmals in ihrer Geschichte von Reichsfeinden erobert. Die Präsenz des Silvanus und seiner Truppen hatte dies im Jahr 354/5 noch verhindert, obwohl nach der Eroberung aller sonstigen römischen Stützpunkte das gesamte niederrheinische Gebiet von den Franken bereits in Besitz genommen worden war. Silvanus' Tod öffnete ihnen faktisch auch die Tore der letzten römischen Festung am Rhein.

Erst zehn Monate später wurde Köln von den Römern zurückgewonnen. Iulian, der Neffe von Constantius II., kam als Caesar nach Gallien und konnte Köln wiedergewinnen; seine praesentia erlaubte zumindest eine gewisse Wiederbelebung der Stadt, wenn auch keine völlige Erholung. Vielleicht hat er auch dafür gesorgt, daß das Gelände zwischen der bisherigen römischen Stadtmauer und dem Rhein damals bereits in den ummauerten Bereich einbezogen wurde. Möglicherweise veranlaßte er auch die Stationierung eines Teils der römischen Rheinflotte in diesem neuen Stadtteil Kölns. ${ }^{72}$ So mochte seine Anwesenheit bei der zahlenmäßig bereits geschrumpften Kölner Bevölkerung wieder einige Hoffnung erweckt haben. Solange Iulian sich in Gallien aufhielt - bis 360 - blieb tatsächlich die Lage am Rhein im wesentlichen ruhig, ja selbst der Abmarsch nach dem Balkan zum Kampf gegen Constantius II. löste keine Einfälle von jenseits der Grenzen aus. Valentinian I. konnte so auf Iulians Werk aufbauen und die

\footnotetext{
${ }^{71}$ Siehe Bleckmann (Anm. 69) 477ff.

${ }^{72}$ Siehe N. Aten - Gjergj Frasheri - F. Kempken - M. Merse, Ausgrabungen auf dem Heumarkt in Köln. Zweiter Bericht zu den Untersuchungen von Mai 1997 bis April 1998, KJb 31, 1998, 481 ff., bes. 488ff.; ferner Hellenkemper (Anm. 29) 52.
} 
Grenze noch weiter befestigen. Fast seine gesamte Regierungszeit von 365375 blieb er in Gallien; auch Köln besuchte er ${ }^{73}$ doch ein längerer Aufenthalt ist nicht mehr nachweisbar. Es gab wichtigere Orte als die so schwer geplünderte Stadt.

Kurze Besuche kann man auch von den folgenden Kaisern vermuten, nachweisen aber läßt es sich für niemanden mehr; doch all dies stand nur noch im Zusammenhang der schnellen Grenzsicherung. Valentinian II., der jüngere Sohn Valentinians I., ist am 2. März 390 in Confluentes (Koblenz) nachgewiesen, ${ }^{74}$ wo er einen Erlaß ausgefertigt hat. Die Reise auf dem Rhein hätte ihn in einem einzigen Tag auch nach Köln führen können. $\mathrm{Ob}$ dies geschehen ist, bleibt offen. Das gilt auch für Eugenius, den von dem Heermeister Arbogast vorgeschobene Usurpator von 392-394. Zwar kennen wir aus Köln noch eine Inschrift, die von der Erneuerung eines größeren Bauwerks spricht. ${ }^{75}$ Diese Maßnahme erfordert jedoch nicht die Anwesenheit des Kaisers selbst. Dennoch ist sie aus der Gesamtsituation heraus sehr wahrscheinlich, nicht weniger als die der ephemeren Herrscher, die sich in den ersten Jahrzehnten des 5. Jh. für jeweils kurze Zeit im nördlichen Gallien am Kampf um die Macht beteiligten: Constantinus III. oder Iovinus. Doch für Köln als römische Stadt war es letztlich ohne Belang, ob der jeweilige Machthaber nahe oder fern war. Realität hatten für die Bewohner Agrippinas nur noch die fränkischen Kleinkönige. Als sich einer von ihnen im ehemaligen Praetorium der römischen Statthalter niederließ, war die Welt verändert. ${ }^{76}$ Nun galt es, sich auf die Nähe des fränkischen Königs einzustellen.

Köln, Januar 2003

\footnotetext{
${ }^{73}$ C. Th. 11, 1, 1; vgl. Seeck (Anm. 48) 31. 234.

${ }^{74} \mathrm{C}$. Th. $10,18,3$ (Seeck).

${ }^{75}$ CIL XIII $8262=$ Dessau $790=$ Galsterer (Anm. 32) Nr. 188. Dazu als letzte umfassende Behandlung Th. Grünewald, Arbogast und Eugenius in einer Kölner Bauinschrift (CIL XIII 8262), KJb 21, 1988, 243ff.

${ }^{76}$ G. Precht, Baugeschichtliche Untersuchungen zum römischen Praetorium in Köln, Köln 1973, 28 ff.
} 